\title{
TIGHT FRAMELET PACKETS ON LOCAL FIELDS OF POSITIVE CHARACTERISTIC
}

\author{
Firdous A. SHAH AND M. YOUNUS BHAT
}

\begin{abstract}
An important tool for the construction of tight wavelet frames on local fields of positive characteristic with the help of unitary extension principles was presented by Shah and Debnath [Tight wavelet frames on local fields, Analysis, 33 (2013), 293-307]. In this paper, we continue the study based on the extension principles and give an explicit construction of a class of tight framelet packets on local fields of positive characteristic.
\end{abstract}

Mathematics subject classification (2010): 42C40, 42C15, 43A70, 11S85.

Keywords and phrases: Wavelet, tight wavelet frame, framelet packet, local field, extension principle, Fourier transform.

\section{REFERENCES}

[1] B. BEHERA AND Q. JAHAN, Wavelet packets and wavelet frame packets on local fields of positive characteristic, J. Math. Anal. Appl. 395 (2012), 1-14.

[2] J. J. BenEDETtO AND R. L. Benedetto, A wavelet theory for local fields and related groups, J. Geom. Anal. 14 (2004), 423-456.

[3] Q. Chen AND Z. ChAnG, A study on compactly supported orthogonal vector-valued wavelets and wavelet packets, Chaos, Solit. Fractals. 31 (2007), 1024-1034.

[4] C. K. CHUi AND C. LI, Non-orthogonal wavelet packets, SIAM J. Math. Anal. 24 (3) (1993), 712 738.

[5] R. R. Coifman, Y. Meyer, S. Quake And M. V. Wickerhauser, Signal processing and compression with wavelet packets, Technical Report, Yale University, 1990.

[6] L. Debnath And F. A. Shah, Wavelet Transforms and Their Applications, Birkhäuser, New York, 2015.

[7] YU. A. FARKOV, Orthogonal wavelets with compact support on locally compact Abelian groups, Izv. Math. 69 (3) (2005), 623-650.

[8] H. K. JiAnG, D. F. Li AND N. Jin, Multiresolution analysis on local fields, J. Math. Anal. Appl. 294 (2004), 523-532.

[9] A. YU. KhrEnNikov, V. M. ShelKovich AND M. SKopina, $p$-Adic refinable functions and MRAbased wavelets, J. Approx. Theory. 161 (2009), 226-238.

[10] W. C. LANG, Orthogonal wavelets on the Cantor dyadic group, SIAM J. Math. Anal. 27 (1996), 305-312.

[11] D. LU AND Q. B. FAN, A class of tight framelet packets, Czechoslovak Math. J. 61 (2011), 623-639.

[12] S. F. LUKOMS KII, Multiresolution analysis on product of zero-dimensional Abelian groups, J. Math. Anal. Appl. 385 (2012), 1162-1178.

[13] S. F. LUKOMSKII, Step refinable functions and orthogonal MRA on Vilenkin groups, J. Fourier Anal. Appl. 20 (2014), 42-65.

[14] D. Ramakrishnan And R. J. Valenza, Fourier Analysis on Number Fields, Graduate Texts in Mathematics 186, Springer-Verlag, New York, 1999.

[15] A. RON AND Z. SHEN, Affine systems in $L^{2}\left(\mathbb{R}^{d}\right)$ : the analysis of the analysis operator, J. Funct. Anal. 148 (1997), 408-447.

[16] F. A. ShaH, Construction of wavelet packets on p-adic field, Int. J. Wavelets Multiresolut. Inf. Process. 7 (5) (2009), 553-565. 
[17] F. A. SHAH, Biorthogonal p-wavelet packets related to the Walsh polynomials, J. Classical Anal. 2 (2012), 135-146.

[18] F. A. Shah And Abdullah, Nonuniform multiresolution analysis on local fields of positive characteristic, Complex Anal. Opert. Theory. (2014) DOI 10.1007/s11785-014-0412-0.

[19] F. A. SHAH AND ABdullah, Wave packet frames on local fields of positive characteristic, Appl. Math. Comput. 249 (2014), 133-141.

[20] F. A. Shah And Abdullah, A characterization of tight wavelet frames on local fields of positive characteristic, J. Contemp. Math. Anal. 49 (6) (2014), 251-259.

[21] F. A. Shah And L. Debnath, p-Wavelet frame packets on a half-line using the Walsh-Fourier transform, Int. Transf. Spec. Funct. 22 (12) (2011), 907-917.

[22] F. A. Shah And L. Debnath, Tight wavelet frames on local fields, Analysis. 33 (2013), 293-307.

[23] Z. SHEN, Non-tensor product wavelet packets in $L^{2}\left(\mathbb{R}^{s}\right)$, SIAM J. Math. Anal. 26 (4) (1995), 10611074.

[24] T. Stavropoulos And M. Papadakis, On the multiresolution analyses of abstract Hilbert spaces, Bull. Greek Math. Soc. 40 (1998), 79-92.

[25] M. H. TAIBLeSon, Fourier Analysis on Local Fields, Princeton University Press, 1975. 